\title{
General Method to Determine Replica Symmetry Breaking Transitions
}

\author{
E. Marinari, * C. Naitza, and F. Zuliani \\ Dipartimento di Fisica and INFN, Universita di Cagliari, Via Ospedale 72, 07100 Cagliari, Italy \\ G. Parisi ${ }^{\dagger}$ \\ Dipartimento di Fisica and INFN, Universita di Roma La Sapienza, P. A. Moro 2, 00185 Roma, Italy
}

M. Picco

LPTHE, Université Pierre et Marie Curie, Paris VI, Université Denis Diderot, Paris VII, Boite 126, Tour 16, ler étage, 4 place Jussieu, F-75252 Paris CEDEX 05, France

\section{F. Ritort ${ }^{\S}$}

Departament de Fisica Fonamental, Facultat de Fisica, Universitat de Barcelona, Diagonal 647, 08028 Barcelona, Spain (Received 3 March 1998)

\begin{abstract}
We introduce a new parameter to investigate replica symmetry breaking transitions using finite-size scaling methods. Based on exact equalities initially derived by F. Guerra this parameter is a direct check of the self-averaging character of the spin-glass order parameter. This new parameter can be used to study models with time reversal symmetry but its greatest interest lies in models where this symmetry is absent. We apply the method to long-range and short-range Ising spin-glasses with and without a magnetic field as well as short-range multispin interaction spin-glasses. [S0031-9007(98)06955-5]
\end{abstract}

PACS numbers: 75.10.Nr, 02.70.Lq, 05.70.Fh, 64.60.Cn

The subject of replica symmetry breaking has become an important issue in statistical physics [1]. Since replica symmetry breaking was proposed a long time ago [2] there have been several new developments concerning spin-glasses as well as their applications in other areas of statistical physics. Setting aside the question whether and how this transition could be observed in real experiments, it is certainly relevant to establish the validity of meanfield theory for spin-glasses when applied to short-range systems. In this context, quite recently a new controversy has appeared on the problem whether self-averageness (i.e., the independence of the order parameter on the microscopic realization of the quenched disorder) is automatically satisfied in short-range systems. While the answer to this question [3] appears to be closely related to the proper definition of the order parameter and how the thermodynamic limit is taken, there are few doubts that non-self-averaging is the crucial signature for a spinglass scenario where replica symmetry breaks. Although there is not definite proof, recent exact results support this assertion [3-5].

The purpose of this Letter is to unambiguously show that indeed replica symmetry (hereafter referred to as RS) breaks in short-range spin-glasses and that the genuine feature of the broken phase relies on the non-selfaveraging character of the order parameter. While the major part of the work in spin-glasses has been focused on models where there is a time reversal symmetry in the Hamiltonian this is not an essential requirement for the existence of a replica symmetry breaking (RSB) transition. In models with time reversal symmetry (hereafter referred to as TRS), RS and TRS break simultaneously at the spin-glass transition temperature. Because both RS and TRS break precisely at the same temperature, it is very difficult to distinguish the different features related to both transitions. Indeed, the main distinction between the droplet [6] and the mean-field approaches relies on which symmetries break at the spin-glass transition temperature. While in the first approach only TRS breaks at the transition temperature, in the second approach both symmetries break. The most widely used parameter to locate spin-glass transitions (the Binder parameter) signals the breaking of time reversal symmetry rather than the other. Consequently, the major part of numerical calculations using the Binder parameter does not show that RS breaks at the spin-glass transition temperature but rather whether TRS breaks. Then, it is essential to look for signatures of replica symmetry breaking in models where time reversal symmetries are lacking.

A large class of models where TRS is not present is spin-glasses in an external magnetic field or multispin $p$-interaction spin-glass models ( $\mathrm{p}-\mathrm{SG}$ ) with $p$ being odd. The first class of models can be described by Hamiltonians of the type

$$
\mathcal{H}=\mathcal{H}_{0}-h \sum_{i} \sigma_{i}=-\sum_{(i, j)} J_{i j} \sigma_{i} \sigma_{j}-h \sum_{i} \sigma_{i}
$$

where the term $h \sum_{i} \sigma_{i}$ breaks the TRS $(\sigma \rightarrow-\sigma)$ of the Hamiltonian $\mathcal{H}_{0}$. On the other hand, models of p-SG take the general form

$$
\mathcal{H}=-\sum_{\left(i_{1}, i_{2}, ., i_{p}\right)} J_{i_{1} i_{2} \ldots i_{p}} \sigma_{i_{1}} \sigma_{i_{2}} \ldots \sigma_{i_{p}}
$$


For $p$ odd, TRS is absent. The interest of this last family of models [contrary to (1)] relies on the fact that there is no parameter which when appropriately tuned restores TRS [this happens in the family of models of Eq. (1), where TRS is recovered if $h=0$ ].

When studying phase transitions in ordered systems, one generally computes the temperature dependence of cumulants of the order parameter distribution such as susceptibilities (second moments) or adimensional parameters such as the kurtosis (Binder parameter) or the skewness of the order parameter distribution. The usefulness of these quantities to distinguish RSB transitions is hampered by the fact that finite-size corrections to the leading scaling behavior of the Binder parameter are big. For RSB transitions it is then convenient to consider adimensional quantities which depend on other genuine features of the transition (and not only on TRS) such as self-averageness. Our purpose here is to define a suitable parameter which is the analog of the Binder parameter for transitions where TRS breaks and which can be used to locate spin-glass transitions where RS breaks. In spin-glasses the order parameter is not the global magnetization but a measure of the freezing of the spins, the Edwards-Anderson parameter $q$ [7]. The appropriate way to compute this parameter is to consider two replicas (i.e., two identical copies of the same system) and compute the overlap, $q=(1 / V) \sum_{i=1}^{V} \sigma_{i} \tau_{i}$, where $V$ is the size or volume of the system. Expectation values of the moments $\left\langle q^{k}\right\rangle_{\mathrm{BG}}$ allow one to reconstruct the order parameter distribution $P_{J}(q)$, where $\langle\ldots\rangle_{\mathrm{BG}}$ stands for the usual Boltzmann-Gibbs average for a given sample. It has been recently shown by Guerra [5] that sample to sample fluctuations of the cumulants of the order parameter distribution $P_{J}(q)$ are Gaussian distributed in the thermodynamic limit. For instance, the following relationship is fulfilled in spin-glasses in the low temperature phase below $T_{c}$ :

$$
G=\frac{\overline{\chi_{\mathrm{SG}}^{2}}-\bar{\chi}_{\mathrm{SG}}^{2}}{\overline{V^{2}\left\langle\left(q-\langle q\rangle_{\mathrm{BG}}\right)^{4}\right\rangle_{\mathrm{BG}}}-\bar{\chi}_{\mathrm{SG}}^{2}}=\frac{1}{3},
$$

where $\overline{(.)}$ means an average over the quenched disorder and $\chi_{\mathrm{SG}}$ (the spin-glass susceptibility) is defined as

$$
\chi_{\mathrm{SG}}=V\left(\left\langle q^{2}\right\rangle_{\mathrm{BG}}-\langle q\rangle_{\mathrm{BG}}^{2}\right) \text {. }
$$

The interest of defining the parameter $G$ is that it vanishes above the transition temperature in the disordered phase where sample to sample fluctuations of $P_{J}(q)$ disappear in the $V \rightarrow \infty$ limit. Consequently, $G$ is a parameter which plays the same role as the usual Binder parameter $g$ in ferromagnets and is given (in the $V \rightarrow \infty$ limit) by $G(T)=(1 / 3)\left[1-\Theta_{H}\left(T-T_{c}\right)\right]$, where $\Theta_{H}$ is the Heaviside theta function. In RSB transitions (3) goes to zero (as the size $V$ increases) as $1 / V$ for $T>T_{c}$ but converges to a finite value for $T<T_{c}$. We expect the critical temperature (where RS breaks) to be signaled by the crossing of the different curves corresponding to different lattice sizes. Furthermore, close to $T_{c}$ it is reasonable to expect $G(T) \sim G(L / \xi)$, where $\xi$ is a correlation length. We stress that the calculation of $G$ is especially useful in models where TRS is absent. In the presence of TRS the usual Binder parameter $g$ can be used to locate the phase transition with much less numerical effort. But the interest of $G$ is that it emphasizes the non-selfaveraging character of the low temperature phase.

To check these predictions we have performed a numerical simulation of the models of the previous type (1) (with and without a magnetic field) and the $p$-spin model (2). All of the simulations use the parallel tempering method, an efficient algorithm to thermalize small samples [8]. We have studied three different models, the meanfield Sherrington-Kirkpatrick (SK) model [9], the four dimensional (4D) Ising spin-glass, and the model Eq. (2) in four dimensions with $p=3$. In this last case, the Hamiltonian is short ranged, there are two spins per site in a 4D simple cubic lattice and the Hamiltonian couples all possible triplets of spins occupying nearest-neighbor sites of the lattice. More precisely the Hamiltonian reads

$$
\begin{aligned}
\mathcal{H}=-\sum_{i=1}^{V} \sum_{\mu=1}^{D}( & J_{(12,1)}^{i, \mu} \sigma_{1}^{i} \sigma_{2}^{i} \sigma_{1}^{i+\mu}+J_{(12,2)}^{i, \mu} \sigma_{1}^{i} \sigma_{2}^{i} \sigma_{2}^{i+\mu} \\
& +J_{(1,12)}^{i, \mu} \sigma_{1}^{i} \sigma_{1}^{i+\mu} \sigma_{2}^{i+\mu} \\
& \left.+J_{(2,12)}^{i, \mu} \sigma_{2}^{i} \sigma_{1}^{i+\mu} \sigma_{2}^{i+\mu}\right)
\end{aligned}
$$

where the pair $(i, \mu)$ denotes the link of the lattice and the spins $\left(\sigma_{1}^{i}, \sigma_{2}^{i}\right)$ occupy the same site $i$ in the lattice.

First, we show the results in the four dimensional Ising spin-glass without a magnetic field $(h=0)$. This is a check of our method since the transition is well known using standard methods [8]. The model is described by Eq. (1) with the $J_{i j}= \pm 1$ connecting nearest-neighbor sites in a cubic lattice of side $L$ with periodic boundary conditions. The simulations were done for sizes $L=4,5,8,10$ (2944, 1920, 1376, 320 samples, respectively) with 100000 Monte Carlo steps (MCS) of thermalization time and the same amount of steps to collect statistics (for $L=10$ we did runs up to 35 million of MCS). Figure 1 shows the results for $G$. Note the existence of a critical temperature above which $G$ goes to zero and below which it converges to $1 / 3$. The different curves cross at a temperature in agreement with that derived from the analysis of the usual Binder parameter [11] and also series expansions [10] $\left(T_{c} \simeq 2.03\right)$.

Next, we consider models without TRS. We first consider the study of the SK model in a magnetic field. The SK model [9] corresponds to Eq. (1) with $J_{i j}$ long-ranged and Gaussian distributed with $\overline{J_{i j}}=0, \overline{J_{i j}^{2}}=1 / V$. The existence of a transition in a field is well established in mean-field theory but there are a few results which corroborate its existence using numerical simulations [12]. Figure 2 shows $G(T)$ for $V=32,256,512,1024$ with $1000,1000,400,150$ samples, respectively. While it is very difficult to see evidence for this transition with the usual cumulants (skewness or Binder parameter) the situation turns out to be clearer with the parameter $G$ where a merging close to $T \simeq 0.6-0.7$ [below $T_{c}(h=0)=1$ ] 


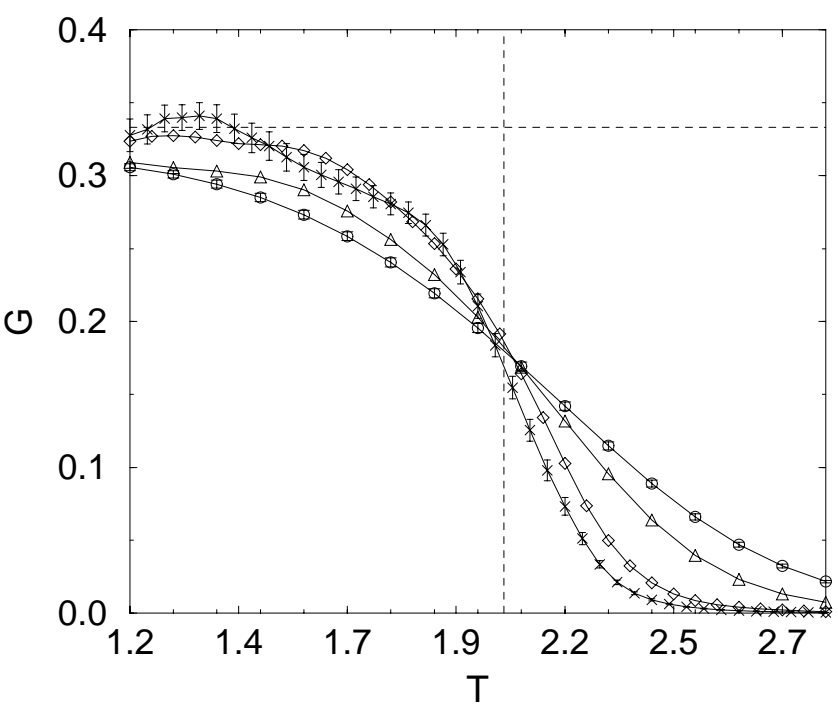

FIG. 1. $G$ in the 4D Ising spin-glass without a field. The horizontal line indicates the expected low $T$ result $G=$ $1 / 3$ while the vertical line indicates the expected transition temperature derived from other methods [10,11]. Error bars are shown for $L=4,10$.

is observed. The figure clearly shows the existence of two temperature regions: a high temperature region where $G$ goes to zero with the volume (as $1 / V$ ) and a low temperature one where $G$ converges to $1 / 3$ (within the precision of the statistics). This shows the existence of the Almeida-Thouless line in the SK model, a result well known in the mean-field theory of spin-glasses but difficult to observe numerically.

The results in the four dimensional Ising spin-glass model in a field are shown in Fig. 3. Simulations were done at a magnetic field $h=0.4$ with statistics ranging from $20000 \mathrm{MCS}$ for $L=3$ up to 450000 for $L=9$.

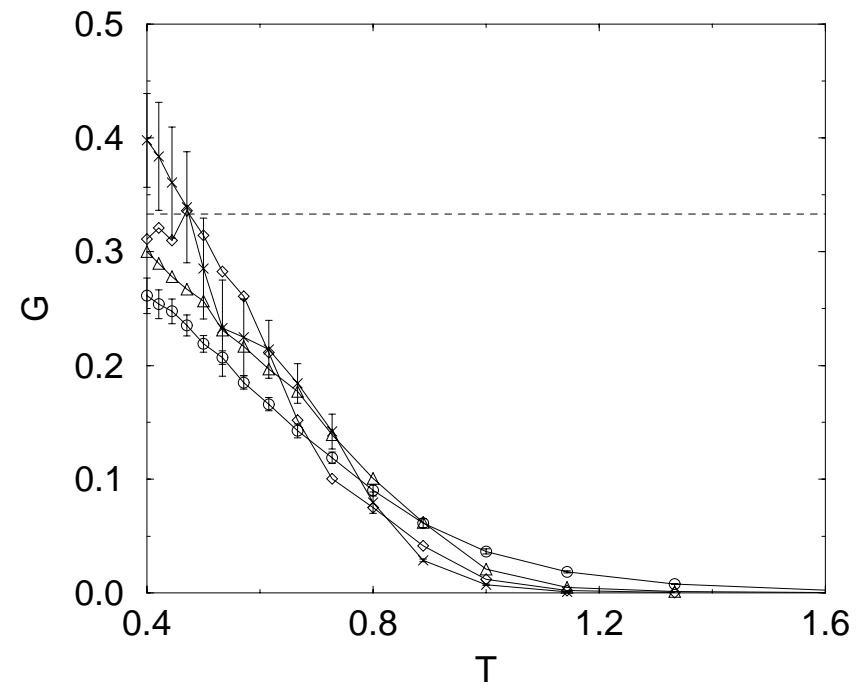

FIG. 2. $G$ in the SK model at $h=0.3$. Error bars are shown for $V=32,1024$. The different curves merge at a temperature well compatible with the theoretical result $T_{c}(h=0.3)=$ $0.65[13]$.

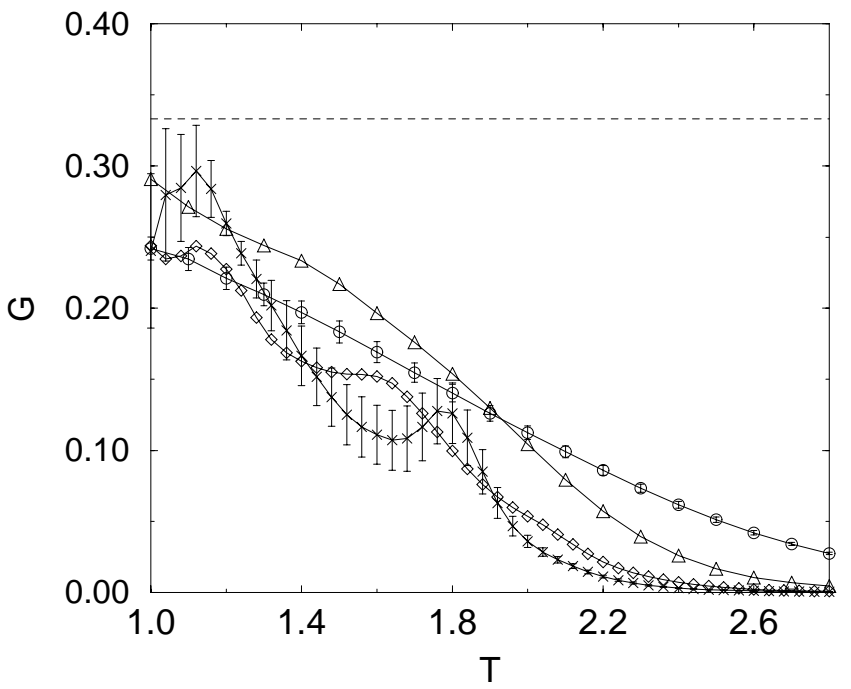

FIG. 3. $G$ in the $4 \mathrm{D} \pm J$ Ising spin-glass at $h=0.4$. The number of samples is 2560,1280,704,64 for $L=3,5,7,9$, respectively. Error bars are shown for $L=3,9$.

We also observe here a behavior very similar to that found in Fig. 2. The existence of the two regions (a high temperature one where $G$ goes to zero and a low temperature one where $G$ converges to a finite value close to $1 / 3$ ) is also clear from the plot.

Figures 2 and 3 show quite unambiguously that there are two regions where self-averaging properties are quite different. This is a strong indication in favor of the existence of a RSB phase transition in spin-glasses in a magnetic field. But a scale invariant crossing point is not as clearly observed in Figs. 2 and 3 compared to what is observed in Fig. 1 for zero magnetic field and Fig. 4 (see below). There are two factors which make numerical simulations of spin-glasses in a magnetic field much more difficult. The first one is related to the difficulty of

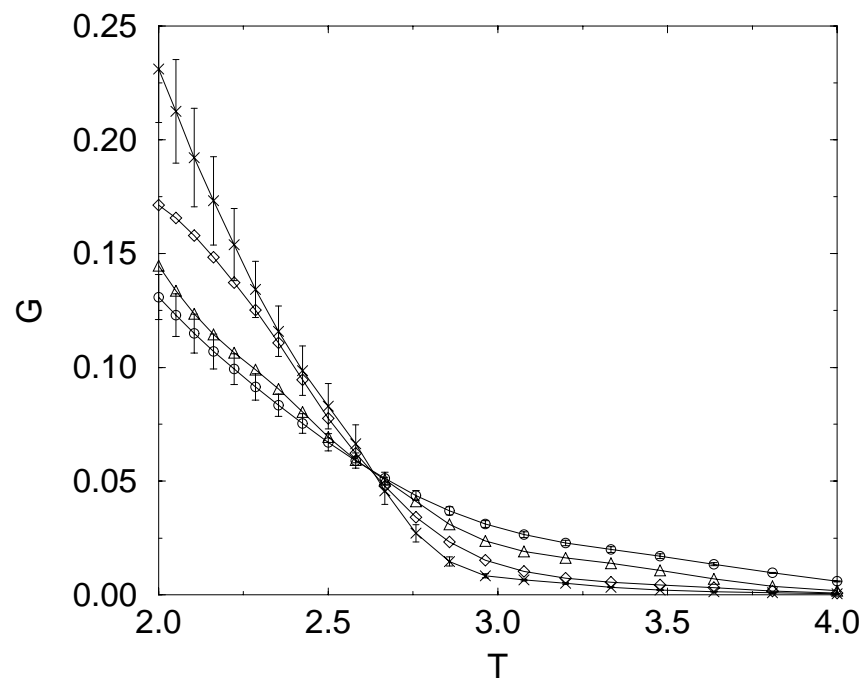

FIG. 4. $G$ in the model (5) without TRS symmetry with threespin interaction and two spins per site. We find that $T_{c} \simeq 2.62$. Error bars are shown for $L=3,6$. 
thermalizing spin-glasses at low temperatures. When the field is switched on, the critical temperature is pushed down (as Figs. 2 and 3 clearly show). This makes thermalization in the low temperature region more difficult. The second factor is related to the fact that Eq. (1) restores the TRS at a zero magnetic field. Consequently, it is natural to expect the existence of a crossover length $L_{c}$ (which increases as the magnetic field decreases) such that above $L_{c}$ the finite-field fixed point dominates the scaling behavior while below $L_{c}$ the scaling behavior is dominated by the zero field fixed point. In the case of Fig. 3 the crossover length was previously estimated $\left(L_{c} \simeq 5[12]\right)$. This crossover effect manifests as a displacement of the crossing point to lower temperatures as the size increases. For large sizes (and always within errors) the crossing point stays at about $T_{c} \simeq 1.2$, a value for the critical temperature which has also been estimated through other methods $[14,15]$.

Assuming that the value of the parameter $G$ at the crossing point corresponds to a universal amplitude, we find (after examination of the data for the SK and the $4 \mathrm{D}$ Ising spin-glass at zero and finite field) that $G_{c}=G\left(T=T_{c}\right)$ clearly increases with the field. This result suggests (in case the previous assumption is correct) that the transition without a field and in a field is determined by different fixed points [16].

To check that the parameter $G$ is indeed a good tool to determine RSB transitions it would be more convenient to consider a model where there is no external small parameter (like the field) which can restore the TRS. For such a model there will not be a crossover length $L_{c}$, and a crossing point for the parameter $G$ should be easier to see already for small sizes. To confirm these expectations we have investigated model (5) with $J^{i, \mu}=$ \pm 1 in four dimensions in lattices of sizes $L=3,4,5,6$ with 100000 MCS of statistics per temperature. The results are shown in Fig. 4. Our results show an algebraic divergence of the spin-glass susceptibility $\chi_{\mathrm{SG}}$, and a leastsquares fit gives $\chi_{\mathrm{SG}} \sim\left(T-T_{c}\right)^{-\gamma}$ with $T_{c} \simeq 2.63$ and $\gamma \simeq 1.0$. This value of $T_{c}$ is in striking agreement with the crossing point observed in Fig. 4. If one assumes, as said before, that $G(T) \sim G(L / \xi)$ with $\xi \sim\left(T-T_{c}\right)^{-\nu}$ then $(d G / d T)_{T=T_{c}} \sim L^{1 / \nu}$. A power law fit yields $\nu \simeq 1 / 2$ suggesting that both $\gamma$ and $\nu$ are close to mean-field values.

Summarizing, we have proposed a new parameter $G$ based on exact inequalities initially derived by Guerra [5]. This parameter is suited to numerically study replica symmetry breaking transitions. The parameter $G$ in Eq. (3) has the good properties of being bounded and positive (a property which does not have the usual Binder parameter used for spin-glasses without TRS) and can be used as a good indicator for RSB transitions using finitesize scaling methods. At high temperatures, $G$ goes to zero as $1 / V$ where $V$ is the volume of the system while at low temperatures it converges to $1 / 3$ in the $L \rightarrow \infty$ limit. We have investigated the $4 \mathrm{D}$ and the SK model in a field finding evidence for an RSB transition. But the nicest application of the method is for models where there is no tunable parameter which restores TRS (like the magnetic field). By introducing a new short-range $p$-spin model [Eq. (5)] we have shown that $G$ is indeed a good indicator for RSB. We have considered a model with $p=3$ in 4D showing that $G$ displays a crossing point where the spinglass susceptibility diverges. Finally, we want to stress that the information gathered from $G$ in models without TRS cannot be extracted in an easy way from the usual standard cumulants of the sample averaged $P(q)$. The genuine property of replica symmetry breaking transitions in disordered systems is the non-self-averaging character of the spin-glass order parameter, a feature which is specifically taken into account within the present method. A deeper understanding of the appropriate renormalization group approach in spin-glasses is certainly needed to clarify the appropriate theoretical framework to deal with these types of phase transitions.

F. R. acknowledges Theo Nieuwenhuizen for discussions and a careful reading of the manuscript.

*Email address: marinari@ca.infn.it

${ }^{\dagger}$ Email address: giorgio.parisi@ roma1.infn.it

\#Email address: picco@lpthe.jussieu.fr

${ }^{\S}$ Email address: ritort@ffn.ub.es

[1] M. Mézard, G. Parisi, and M. A. Virasoro, Spin Glass Theory and Beyond (World Scientific, Singapore, 1987); K. Binder and A.P. Young, Rev. Mod. Phys. 58, 801 (1986); K.H. Fischer and J. A. Hertz, Spin Glasses (Cambridge University Press, Cambridge, England, 1991).

[2] A. Blandin, J. Phys. (Paris), Colloq. 39, C6-1499 (1978); A. J. Bray and M. A. Moore, Phys. Rev. Lett. 41, 1068 (1978); G. Parisi, J. Phys. A 13, 1101 (1980); 13, 1887 (1980).

[3] C. M. Newman and D. L. Stein, Phys. Rev. Lett. 76, 515 (1996).

[4] M. Aizenman and P. Contucci, cond-mat 9712129.

[5] F. Guerra, Int. J. Mod. Phys. B 10, 1675 (1996).

[6] D. S. Fisher and D. A. Huse, Phys. Rev. B 38, 386 (1988); Phys. Rev. B 38, 373 (1988).

[7] S.F. Edwards and P.W. Anderson, J. Phys. F 5, 965 (1975).

[8] See the article review by E. Marinari, G. Parisi, and J. J. Ruiz-Lorenzo, in Spin Glasses and Random Fields, edited by A. P. Young (World Scientific, Singapore, 1997).

[9] D. Sherrington and S. Kirkpatrick, Phys. Rev. Lett. 35, 1792 (1975).

[10] R. Singh and S. Chakravarty, Phys. Rev. Lett. 57, 245 (1986).

[11] D. Badoni, J.C. Ciria, G. Parisi, J. Pech, F. Ritort, and J. J. Ruiz-Lorenzo, Europhys. Lett. 21, 495 (1993).

[12] M. Picco and F. Ritort, J. Phys. I (France) 4, 1619 (1994).

[13] J. R. de Almeida and D. J. Thouless, J. Phys. A 11, 983 (1978).

[14] M. Picco and F. Ritort, cond-mat 9702041.

[15] E. Marinari, G. Parisi, and F. Zuliani, cond-mat 9702253; cond-mat 9802224.

[16] A. J. Bray and S. A. Roberts, J. Phys. C 13, 5405 (1980). 